\title{
ESTUDIO BIOECONÓMICO EN EL ESTABLECIMIENTO DE UNA EXPLOTACIÓN CAPRINA EN COSTA RICA ${ }^{1}$
}

\author{
David Mora-Valverde ${ }^{2}$
}

\begin{abstract}
RESUMEN
Estudio bioeconómico en el establecimiento de una explotación caprina en Costa Rica. Se desarrolló un proyecto caprino en la provincia de Cartago, Costa Rica, entre los meses de setiembre del 2007 hasta enero del 2009, el cual tuvo como objetivo ejecutar un sistema práctico para determinar datos productivos y financieros por concepto de lechería bajo un determinado sistema integral. Lo anterior implicó el diseño y aplicación de técnicas referentes a manejo zootécnico-ambiental, recurso animal, forrajes, agronomía, administración y proyección financiera. Los resultados económicos arrojan una inversión de $\varnothing 14305000$ (570 colones por dólar) para una unidad con 60 hembras con un costo de producción por litro de leche de $\varnothing 459$, con una utilidad base del $18 \%$. Financieramente la proyección del proyecto generó un valor actual neto (VAN) de $\varnothing 13700$ 000, con un periodo de vida útil del mismo estimado en diez años. Conjuntamente se determinó un valor de Tasa interna de retorno (TIR) de $26,23 \%$ por concepto de venta de leche únicamente. Respecto a los costos productivos, el rubro de mayor importancia representó la adquisición del alimento concentrado, con un $41 \%$ del costo total de producción, seguido por la mano de obra $(22 \%)$ y la mensualidad del crédito solicitado (17\%). El dato de rentabilidad económica correspondió a un $15 \%$.
\end{abstract}

\begin{abstract}
Bio-economic study for establishing of a goat farm in Costa Rica. A goat project was developed in the province of Cartago, Costa Rica, from September 2007 through January 2009, with the objective of implementing a practical system to determine productive and financial data for dairy production under a given integrated system. This involved the design and application of techniques related to livestock and environmental management, animal resources, feed, agronomy, administration and financial projections. The economic results showed a investment of $\varnothing 14305000$ (570 colones per dollar) for a 60 female goat unit with a production cost per liter of milk of $\varnothing 459$, with utilities of $18 \%$. Financially, the projection generated a net present value (NPV) of $\varnothing 13700$ 000, with a lifetime estimated in ten years. Simultaneously, a value of Internal Rate of Return (IRR) of $26.23 \%$ from the sale of milk was determined. Regarding production costs, the most important item represented the acquisition of the concentrate, with $41 \%$ of the total production cost, followed by labor $(22 \%)$ and the monthly payment for the credit requested (17\%). The economic profitability was $15 \%$.
\end{abstract}

Key words: Goats, dairy science, sustainability, profitability, agro business.

Palabras clave: Caprinos, lechería, sostenibilidad, rentabilidad, agronegocios.

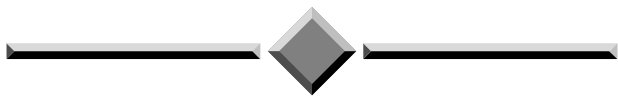

1 Recibido: 22 de octubre, 2009. Aceptado: 17 de mayo, 2010. Esta investigación pertenece al proyecto 737-A8-155. Inscrito ante la Vicerrectoría de Investigación, Universidad de Costa Rica.

2 Estación Experimental Alfredo Volio Mata. Facultad de Ciencias Agroalimentarias. Universidad de Costa Rica. Cartago, Costa Rica. david. mora@ucr.ac.cr 


\section{INTRODUCCIÓN}

La leche de cabra y sus derivados son productos que representan un papel significativo, económico y nutricional (Corrales y Chacón 2005) en los mercados donde históricamente es uno de los componentes principales de la dieta de millones de personas. El carácter globalizado de los actuales mercados permite efectuar explotaciones capaces de suplir la demanda de mercados exigentes y distantes tal y como ya se da en Costa Rica con diversas marcas de derivados lácteos y cárnicos. En el caso de la caprinocultura, cabe recalcar que en Costa Rica, ha sufrido desde sus inicios importantes problemas de desorganización y con escasas excepciones, no ha logrado distinguir la importancia de una organización comercial en la cual se consoliden estrategias de posicionamiento de los diferentes tipos de derivados de la leche, que utilicen como estrategia, la máxima proyección de sus pocas cualidades en el mercado nacional, sin excluir al mercado internacional.

El desarrollo de este tipo de estrategias debe ir atado a un conocimiento primario en el cual se tengan claras las diferentes variables que inciden sobre la lechería caprina en condiciones costarricenses, incluyendo los costos atribuibles a la obtención de cada unidad de materia prima para las plantas de procesamiento de leche, los cuales dependen naturalmente de los factores productivos del empresario cabrero para que una explotación de esta naturaleza sea rentable. Lo anterior se sustenta en que el caprinocultor novel nacional cuenta con una plataforma de información de costos desactualizada, dispersa y de relativa confianza, que recabarla le genera un gasto importante en dinero y tiempo que bien podrían ser aprovechados en el dominio de los factores productivos primarios (reproducción, nutrición, manejo, etc.) de su futura o actual explotación. Este factor informativo puede acarrearle facilidades en la toma de decisiones para incursionar en proyectos potencialmente rentables.

El presente estudio tuvo por objetivo guiar al profesional en producción animal, al productor caprino y/o al iniciador en la caprinocultura a través de una esquematización de los diferentes rubros para establecimiento y operación, la mayoría indispensables para sobrevivir en el negocio caprino, basado en una explotación de carácter mediano (60 cabras adultas) bajo un sistema descrito de manejo. Con ésto, se espera ofrecer un marco de referencia para identificar los costos del precio de la leche caprina, bajo una estructura austera donde se toma en cuenta el respeto al medio ambiente y al bienestar animal, que sirva como modelo empresarial para la capacitación y el mejoramiento de la transferencia de conocimientos en caprinocultura.

\section{MATERIALES Y MÉTODOS}

La investigación perteneció al proyecto "Análisis Bioeconómico para el establecimiento de explotaciones caprinas" ligado a la Estación Experimental Alfredo Volio Mata de la Universidad de Costa Rica en Ochomogo, Cartago y se implementó en la misma provincia, específicamente en el sector conocido como Avance, a una altura de $1750 \mathrm{msnm}$ con una temperatura media anual de $18,5^{\circ} \mathrm{C}$ y una precipitación media mensual de $2150 \mathrm{~mm}$. Fue efectuado entre los meses de setiembre del 2007 hasta enero del 2009. Éste proyecto nació a partir de la creación una unidad productiva primaria la cual, entre otras, debía proveer una cuota específica de materia prima láctea a una empresa emergente dedicada al proceso y comercialización de lácteos caprinos. Para ésto, se determinaron los factores necesarios para la autosostenibilidad y rentabilidad del negocio en el tiempo. Lo anterior incluyó desde el diseño hasta la aplicación de diversas técnicas referentes a manejo zootécnico y ambiental, recurso animal, forrajes, agronomía y administración, para así contemplar el proyecto desde una perspectiva integral. Los índices productivos y reproductivos presentados en este documento, con los recursos y tecnología propuestos, son potencialmente factibles en la zona, demostrados repetidamente por otras unidades en la región del proyecto.

\section{Detalles generales del módulo productivo}

Plan general. Se identificaron las necesidades para el establecimiento de la empresa y se ubicó la unidad productiva primaria en las cercanías de la planta procesadora. El proyecto está situado en las cercanías de Tres Ríos, Avance y Dulce Nombre de Cartago para localizar una finca que estuviese disponible para alquiler con buen potencial para la producción de forrajes, el mantenimiento de los animales y con accesibilidad bajo la modalidad de alquiler. Se concretó utilizar 
una finca disponible en Avance de Cartago cercana a la planta de procesamiento de lácteos, de extensión suficiente, la cual contó con las necesidades básicas para establecer el proyecto. El objetivo de la empresa una vez estabilizada fue inicialmente contar con una población de 60 animales. De éstos, se proyectó como objetivo de producción, un $60 \%$ constante de animales productivos. Se caracterizaron los factores de estudio como: a. Alimentación, b. Producción, c. Manejo, d. Manejo reproductivo e. Mano de obra, f. Infraestructura, g. Factibilidad económica.

a) Aseguramiento del factor alimenticio. $\mathrm{Se}$ realizó una evaluación del componente forrajero y las necesidades según la población proyectada, lo cual implicó para el tamaño de hato definido, una cantidad diaria mínima de $250 \mathrm{~kg}$ de forraje fresco (o en su defecto $65 \mathrm{~kg}$ de materia seca, aproximadamente) ofrecido en dos turnos al día. Para ello se contempló el establecimiento de pasturas de corte (Pennisetum purpureum y Sorghum almum) con las cuales se aumentase la capacidad forrajera por área en comparación a sistemas de pastoreo. Una hectárea y media de pasto de corta se estimó para manejar suficiente material alimenticio. Asimismo, la extensión de la finca escogida permite un excedente en épocas críticas con pasto kikuyo (Pennisetum clandestinum) ya establecido el cual se aprovecharía bajo la modalidad de corta. Para ésto, se adquirieron los equipos necesarios de apoyo a las actividades de alimentación animal (corta y picado). La formulación de las raciones se efectuó con un programa específico para tal fin. El rubro factor alimenticio incluyó la transformación de los desechos caprinos en abono orgánico para uso de la misma unidad forrajera.

b) Producción. El programa inicial de trabajo tuvo como objetivo, según solicitud de la planta industrializadora a proveer, un aproximado de 400 litros semanales con los cuales se optimizaría la capacidad procesadora de ésta. Para llegar a este objetivo el cual fue superior a la realidad genética de los animales disponibles y adquiridos para el proyecto, se estimó un año de adaptación dentro del cual se efectuaría una selección genética (compra y reventa de animales) que permitiese mejorar y pulir el hato de manera que el volumen requerido se lograse a través de una cantidad menor de animales con genética superior. La leche se comercializó fresca y se entregaba a la planta de procesamiento con una frecuencia de cuatro días por semana. Se incluyó un sistema de ordeño manual. c) Manejo. La unidad debía funcionar dentro del margen de respeto al comportamiento animal, ya que por iniciativa del órgano ejecutor se definió el contar con los estándares mínimos (HFAC 2005) en cuanto a este tema se refiere. En el sistema de semiestabulación, se contempló que los animales recibiesen su ración dos veces al día en las instalaciones bajo techo (encierros) y dependiendo del estado del tiempo las cabras accesaban a corrales externos. Lo anterior para permitir que el animal cambie de un entorno de alta densidad animal a otro de leve, donde pueda ejercitarse de manera controlada para el productor.

d) Manejo reproductivo. Para éste se definió dividir el hato de hembras en cinco grupos productivos, controlados reproductivamente mediante la sincronización hormonal y con monta natural de dos machos raza Saanen. Lo anterior para que en el debido momento de estabilización reproductiva se minimicen los picos productivos generalizados por esta especie en nuestras latitudes que corresponden a los primeros meses del año (diciembre, enero y febrero) y a los meses de junio, julio y agosto, donde existe siempre sobreoferta de leche.

e) Mano de obra. Para la ejecución del estudio se calculó la mano de obra necesaria, determinando que 1,5 jornales cubre los requisitos de trabajo para el manejo de un hato de 75 cabras. Para ello se estudió el ciclo de labores de la Estación Experimental Alfredo Volio Mata y de otras unidades semejantes en la zona de Turrialba y Cartago. Lo anterior facilitó hacer una distribución de jornadas semanales para un mejor aprovechamiento del recurso humano. Se definieron las tareas de ejecución, composición y dedicación de tareas de este recurso como asalariado permanente (peón o peones que realizan el mantenimiento de las instalaciones así como el manejo de animales y ordeño (Sabadzija et al. 2007). Las tareas incluidas dentro del cálculo laboral fueron: la ordeña, el manejo sanitario, control reproductivo, corta y acarreo del forraje, mantenimiento de la calidad de la leche, higiene y ornato de la cabreriza, tratamiento de los desechos (volteos, acarreo), fertilización de pasturas y mantenimiento general de la unidad y el mantenimiento de registros en los anteriores rubros.

f) Infraestructura. La unidad caprina inició su construcción en enero del 2008 y operó bajo el sistema de confinamiento acorde con estándares internacionales para caprinos y ovinos (HFAC 2005) utilizando para ello áreas de confinamiento total así como áreas 
de esparcimiento (patios de ejercicio). Se fabricaron corrales y cepos desarmables de metal (Luttman 1986, Simiane y Le Mens s.f.) supervisando su construcción. El área techada consistió en un galpón de $250 \mathrm{~m}^{2}$ con la instalación de seis corrales desmontables metálicos con capacidad para albergar doce animales cada uno (densidad de $1,5 \mathrm{~m}^{2}$ por animal), una bodega/oficina, sala de ordeño y habitación para el trabajador(es) encargado. Se definió el requerimiento de siete encierros separados para discriminar entre grupos de cabras (preñadas, secas, lactantes y crías). Así como de una pequeña sala de ordeño. Se supervisó la construcción de este módulo hasta su conclusión.

g) Potrero de esparcimiento. Estos permiten que los animales tengan acceso a ciertas horas del día, con una densidad estimada de cinco metros cuadrados por animal $\left(250 \mathrm{~m}^{2}\right.$ en total). No se tomó en cuenta el aporte alimenticio de estos potreros para el cálculo nutricional, debido a que su fin no es proveer alimento.

h) Factibilidad económica. Los datos utilizados para el ejercicio de costos de establecimiento fueron tomados desde noviembre del 2007 hasta febrero del 2009 , los precios de mercado fueron actualizados hasta febrero del 2009 y los datos de producción lechera se obtuvieron entre febrero y octubre del 2008. Para los cálculos se incluyeron todos los costos atribuibles a la construcción de la unidad caprina, así como lo referente a la producción agrícola del forraje, alquiler del terreno, mano de obra, sanidad de los animales, identificación, reproducción, mantenimiento, conservación de la leche, habitación del encargado, energía y mensualidad del crédito solicitado. La venta oportuna de genética, carne de cabrito lechal y abonos orgánicos pueden generar ingresos paralelos, pero no fueron considerados para la presente evaluación de factibilidad.

Para estimar la evolución de la unidad caprina y de la producción de leche, el progreso de estos rubros se expresa en litros promedios diarios y litros totales anuales estimados. Este progreso es utilizado para la evolución de los ingresos en la evaluación financiera.

Los cálculos económicos se efectuaron a través de la metodología INTA (Sabadzija et al. 2007), donde se definieron, cuantificaron y valoraron los bienes de capital agrario requeridos para el modelo propuesto. Posteriormente, proyectando a un periodo de diez años, se estimaron los resultados económicos potenciales de la actividad lechera, no así las complementarias (abono orgánico, cría) mediante el margen bruto (Ingresos brutos - costo directo); el costo de producción total y por litro de leche. El resultado económico del establecimiento como conjunto de proyecto se calculó mediante el valor de Ingreso neto (IN) anual y la rentabilidad (IN/KT) y la evaluación de la inversión se estimó (a diez años, sin crecimiento del hato) mediante el valor actual neto (VAN) y la tasa interna de retorno (TIR) utilizando el método de tanteos (Benavides 2007), donde se definió anualmente un incremento en los costos de producción de un 10\% igualando en la misma escala el precio de venta de la leche.

\section{RESULTADOS Y DISCUSIÓN}

El análisis teórico para evaluar las posibilidades de un proyecto permite que el mismo pueda estar apoyado en indicadores técnicos (Nastasi 2007). Concibiendo un proyecto como un agroecosistema, este es la unidad de análisis, y su contexto, donde se observan la totalidad de factores que lo integran para poder llegar a una descripción de la autonomía de un proyecto (Vilaboa et al. 2006). Junto a ésto, el plan estratégico, como motor de todo el proceso de planificación y también operativo, indica la vía a seguir para formalizar las distintas estrategias y luego verificar y rendir cuentas de su factibilidad (Nastasi 2007).

El flujo de caja anual desarrollado tomó en cuenta todos los factores zootécnicos relevantes para hacer éste lo más cercano a la realidad práctica proyectada (Cuadro 1). Lo anterior definió la inversión requerida, el capital de trabajo, la estimación de producción mensual lechera según el tamaño del hato, los requerimientos de forrajes y mano de obra, manejo sanitario, así como todas las consideraciones respecto al manejo tecnológico para la conservación de la leche. Este sirvió como apoyo para realizar un análisis de sensibilidad en el cual se pudo valorar diferentes escenarios (cambios en: precios de venta unitarios (colones/litro), volúmenes de producción y cuotas del financiamiento) que a su vez modificaron los principales indicadores del proyecto (utilidad neta, tasa interna de retorno, valor presente neto). El plan de inversiones ejecutado para la explotación se presenta en el Cuadro 1 y Cuadro 2.

La propuesta teórico-financiera, presentada ante los interesados, generó respuestas positivas sobre el proyecto, el cual tuvo el visto bueno de especialistas consultados incluyendo autoridades bancarias que aceptaron financiar el mismo. Con lo anterior se logró 
Cuadro 1. Flujograma financiero (en colones) proyectado a cinco años. Cartago, Costa Rica. 2009.

\begin{tabular}{|c|c|c|c|c|c|}
\hline Rubro anual & $\begin{array}{c}\text { AÑO } 1 \\
\text { (2008) }\end{array}$ & AÑ̃ 2 & AÑ̃ 3 & AÑO 4 & AÑO 5 \\
\hline Egreso anual del préstamo & 2085978 & 2085978 & 2085978 & 2085978 & 2085978 \\
\hline Concentrado/hembra lactante & 4070543 & 4477597 & 4.925357 & 5417893 & 5959682 \\
\hline Conc/hembras no lactantes & 666521 & 666521 & 666521 & 666521 & 666521 \\
\hline Salarios & 2646000 & 2910600 & 3.201660 & 3521826 & 3874008 \\
\hline Alquiler finca & 1200000 & 1380000 & 1.587000 & 1825050 & 2098807 \\
\hline Manejo sanitario & 229757 & 252733 & 278006 & 305807 & 336388 \\
\hline Mejoramiento genético & 200000 & 220000 & 242000 & 266200 & 292820 \\
\hline Mantenimiento & 600000 & 660000 & 726000 & 798600 & 878460 \\
\hline Agua/Luz/Teléfono & 180000 & 198000 & 217800 & 239580 & 263538 \\
\hline Gastos varios & 175000 & 192500 & 211750 & 232925 & 256217 \\
\hline Total Gastos & 12053801 & 13043931 & 14142074 & 15360382 & 16712422 \\
\hline \multicolumn{6}{|l|}{ Leche } \\
\hline \# cabras & 60 & 60 & 60 & 60 & 60 \\
\hline Precio / litro (18 \% utilidad) & 541 & 595 & 654 & 720 & 792 \\
\hline$\%$ cabras lactando & $60 \%$ & $60 \%$ & $60 \%$ & $60 \%$ & $60 \%$ \\
\hline Litros leche & 26280 & 26280 & 26280 & 26280 & 26280 \\
\hline Valor leche & 14217480 & 15639228 & 17203150 & 18923465 & 20815812 \\
\hline
\end{tabular}

contar con una guía para el proceso de crecimiento y desarrollo que la unidad caprina debía seguir con el paso del tiempo (crecimiento y mejoramiento de la producción y hato). Una vez implementado el proyecto, los resultados de inversión encontrados se comportaron de manera muy similar a la estimación teórica.

Definida la necesidad productiva y planificada la adquisición de genética caprina, se abocó a la búsqueda de hembras preñadas en diversas zonas del país. Lo anterior permitió tener una perspectiva real de la situación del caprinocultor costarricense, donde las unidades productivas son considerablemente pequeñas y con un enfoque principalmente de pasatiempo o entrada económica extra, reflejando la gran atomización de pequeños productores principalmente de traspatio. Similar a los que indica Velasco et al. (SF) quienes asocian esta actividad a sectores marginales y mayoritariamente de autoconsumo. En relación a los costos de cada una de las inversiones (Figura 1), la compra de vientres y las instalaciones, representaron prácticamente la mitad del total invertido en el proyecto, seguido del derecho de recepción de leche en la planta procesadora (24\%).

La confección del proyecto técnico, la construcción y el montaje y la puesta en marcha de la unidad caprina, contando con el capital de inversión vía crediticia, implicó un costo inicial por periodo de inmovilización de recursos (Castro 2001) relativamente bajo, de un año máximo, tomando en cuenta los detalles zootécnicos de inversión mencionados, ésto permitiría madurar económicamente la unidad y permitir una corriente financiera de liquidez en menor tiempo.

Los cálculos de proyección financiera incluyeron un crecimiento nulo anual del tamaño del hato, manteniéndose constante en 60 vientres, mejorando únicamente su calidad genética, contemplada en el rubro "mejoramiento genético" el cual consiste en la compra anual de animales superiores y descarte de animales de calidad no deseada.

El monto de inversión planificado fue cubierto por un crédito de 15 millones de colones al 11\% de interés anual durante un lapso definido de 15 años. Tomando 
Cuadro 2. Inversiones planificadas y ejecutadas (en colones) en el proyecto de establecimiento de la unidad caprina. Cartago, Costa Rica. 2009.

\begin{tabular}{lrrr}
\hline Rubro & \multicolumn{1}{c}{$\begin{array}{c}\text { Gasto } \\
\text { planificado }\end{array}$} & \multicolumn{1}{c}{$\begin{array}{c}\text { Gasto } \\
\text { ejecutado }\end{array}$} & Diferencia \\
\hline 60 cabras & 4800000,00 & 2985000,00 & 1815000,00 \\
Asociación* & 3400000,00 & 3400000,00 & 0,00 \\
Equipo ordeño & 500000,00 & 1000000,00 & $(500000)$ \\
Tanque de frío & 500000,00 & 500000,00 & 0 \\
Cabreriza** & 2000000,00 & 2250000,00 & $(250000)$ \\
Gastos varios & 200000,00 & 600000,00 & $(400000)$ \\
Herramientas & 900000,00 & 800000,00 & 100000 \\
Habitación & & & 50000 \\
trabajador & 450000,00 & 500000,00 & 0 \\
Pastura corte & 550000,00 & 550000,00 & 000 \\
Corrales & 1000000,00 & 720000,00 & 280000 \\
Capital Trabajo & 1000000,00 & 1000000,00 & 0 \\
Total & 15300000,00 & 14305000,00 & $\varnothing 995000,00$ \\
\hline
\end{tabular}

* Para la planificación de la inversión, en este proyecto se contempló la adquisición de un contrato de asociación con la planta industrializadora como aseguramiento del recibo de la leche, asimismo la unidad caprina participaría como socia de la misma. Para efectos de resultados, este rubro se adjuntó a la mensualidad del crédito obtenido. $* * E l$ factor terreno fue cubierto en el proyecto mediante la figura de alquiler, la cual representó un costo fijo de producción.

en cuenta este valor, se obtuvo uno actual neto (VAN) de $\varnothing 13700$ 000, con un periodo de vida útil del mismo estimado en diez años. Conjuntamente se determinó un valor de tasa interna de retorno (TIR) de $26,23 \%$, que al igual que en el VAN, el flujo por la venta de leche fresca es el responsable único para este ejercicio. Para efectos de caprinocultores que puedan asegurar el ingreso de sus productos al mercado sin necesidad de asociarse a una planta procesadora, los pronósticos de rentabilidad y tasa de retorno no necesariamente se verían mejorados por una disminución en la inversión inicial en relación al no pago por un tipo de asociación. Para estos casos, dentro de la estructura de costos, debe incluirse los incrementos en los gastos de venta atribuibles ya sea al transporte y/o mercadeo de la leche y/o sus derivados.

Del costo de producción de leche obtenido, el rubro de mayor importancia en éste resulta la adquisición

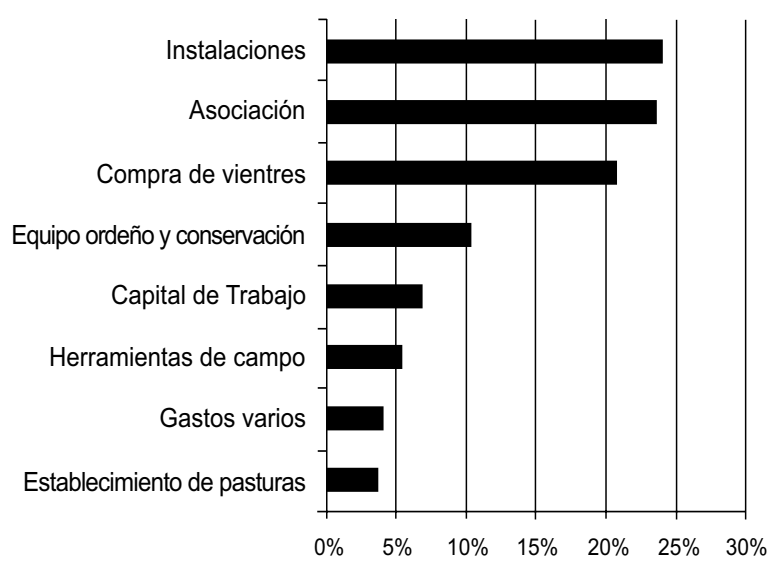

Figura 1. Distribución porcentual de las inversiones ejecutadas en el proyecto unidad caprina. Cartago, Costa Rica. 2009. Cartago, Costa Rica. 2009.

del alimento concentrado, con un $41 \%$ del costo total de producción, seguido por la mano de obra $(22 \%)$ y la mensualidad del crédito (17\%). Lo anterior refleja el poco grado de autonomía alimentaria que caracterizan a las unidades productivas lecheras en Costa Rica. El valor mínimo para producir un litro de leche con las condiciones establecidas fue de $\varnothing 459$, valor en el cual la tasa interna de retorno se acerca más a cero. Para llegar a los datos obtenidos de VAN y TIR se sugirió un margen mínimo de $18 \%$ por utilidad de venta de leche, el cual es un dato razonable (inferior, inclusive) según un sondeo realizado en microempresas caprinas del país. Estos datos concuerdan con Sabadzija et al. (2007), quien reporta en sus resultados al establecer una unidad caprina, un valor de TIR de $18 \%$ con una tasa de interés mayor (12\%), pero toma en cuenta los ingresos brutos percibidos por la actividad de venta de cabritos lechales y animales reproductores. Asímismo Velasco et al. (SF), comparando tres situaciones financieras en lecherías caprinas chilenas obtuvo datos de TIR que fluctuaron entre 16 a $3 \%$. Cabe recordar que para el presente estudio, no se tomó en cuenta los posibles ingresos extra por genética animal, cabrito lechal y/o abono orgánico. De incluirse, probablemente los resultados teóricos hubiesen sido superiores.

Para el caso de otros rubros, Vilaboa et al. (2006) revela en sistemas ovinos lecheros mexicanos un porcentaje de costos por manejo sanitario de un 5\% y respecto a la mano de obra para éstos representa un $26,2 \%$. En comparación al presente estudio se obtuvo un $3 \%$ 
relacionado al manejo sanitario y un $22 \%$ en relación a la mano de obra (Figura 2). El factor autonomía laboral (horas hombre realizadas por los dueños del proyecto) no representó un factor determinante para la viabilidad del proyecto en comparación a lo sugerido por los autores mencionados anteriormente, quienes consideran ésta determinante para el éxito de un emprendimiento ovino-caprino. Lo anterior puede deberse principalmente a los precios de venta del producto final donde al ser una actividad de más difusión con tamaño de unidades superiores y una cantidad superior de éstas (competencia y mayor mercado), obligan la baja en el precio por unidad producida. No así en Costa Rica donde, como se mencionó anteriormente, los productos caprinos son considerados como "accesorios alimentarios", que como parte de la dieta del costarricense. Lo anterior aunado a lo que consideran Chacón et al. (2008), donde se afirma que en Costa Rica es necesario una mayor producción y mercadeo del producto; no solo para modificar la percepción sensorial que de la misma se tiene, sino también para incrementar su consumo.

Respecto al análisis de rentabilidad, éste se convierte en un indicador fundamental para evaluar la eficiencia y eficacia económica de la unidad productiva y posteriormente para la implementación de estrategias que contribuyan a mejorar dicho sistema (Obando 2005). En este caso se analiza la rentabilidad, entendiéndose ésta como la relación (tasa) que compara la utilidad con un aporte de fondos, por lo que se expresa en términos de porcentaje. Para este caso se estimó la rentabilidad desde dos perspectivas, la primera

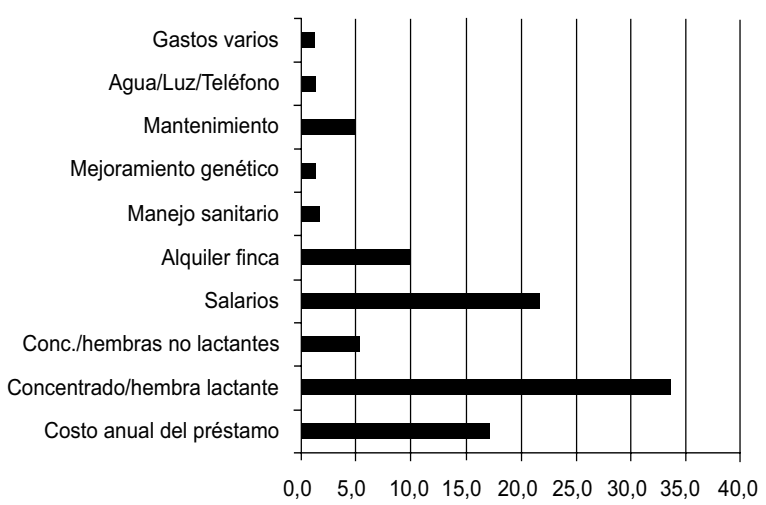

Figura 2. Distribución porcentual de los costos anuales en el proyecto unidad caprina. Cartago, Costa Rica. 2009. fue ésta sobre ventas, la cual toma como premisa el ingreso y su relación con las ventas, y la rentabilidad económica, la cual relaciona el monto de inversión con los ingresos.

Rentabilidad sobre ventas anual:

$\frac{\text { Utilidad anual por ventas : }}{\text { Costo anual }} 15,2 \%$

Rentabilidad económica anual año uno:

\section{Utilidad anual por ventas: $15,1 \%$ Inversión total}

Para los resultados obtenidos se empleó el porcentaje mínimo de utilidad mencionado anteriormente de $18 \%$ en el precio del litro de leche. Lo anterior es poco frecuente en relación a la realidad del mercado nacional donde el precio de venta de la leche se acerca a márgenes de utilidad que rondan el 25 al 30\% respecto al costo calculado en el presente ejercicio.

Al evaluar la potencialidad en casos donde se cuente con tierra disponible para la ejecución de un proyecto de este tipo, así como de una instalación con la cual no sea necesario iniciar a partir de cero un proyecto, los valores de costo de producción y proyecciones financieras podrían arrojar datos más positivos debido a que, entre otros, el aporte correspondiente al pago de un crédito sería menor, en comparación a como se contempla en el presente análisis donde no se cuenta con el rubro terreno.

La principal limitante zootécnica para un emprendimiento en caprinocultura es el acceso a genética de calidad (compra y/o importación de animales vivos, semen, etc.), aspectos que dificultan la mejora productiva en tiempos más cortos.

La producción caprina en Costa Rica y bajo las condiciones expuestas, representa una oportunidad de emprendimiento atractiva siempre y cuando se asegure de previo la incursión en un mercado. Ésta se encuentra en una etapa incipiente que requiere de mecanismos facilitadores que permitan una incursión en el mercado más sólida respecto a iniciativas del pasado. Entre éstos, se debe facilitar a nivel estatal el desarrollo de grupos organizados, la transferencia eficaz de tecnología a nivel institucional y el acceso al financiamiento, factores que, entre otros, estimularían la correcta incursión 
en mercados donde se incluya la explotación animal sostenible y sustentable, que implique beneficios económicos y el desarrollo de capacidades nacionales para el sustento de comunidades locales y la gestión de tecnologías amigables con la naturaleza.

\section{LITERATURA CITADA}

Benavides, H. 2007. Aplicación de métodos numéricos en el análisis financiero, determinación de la TIR por el método de Newton Raphson (en línea). Universidad Técnica Particular de Loja, Ecuador. Consultado feb. 2009. Disponible en http://www.utpl.edu.ec/ucg/images/stories/papers/numericos\%20tir.pdf

Castro, M. 2001. El Valor Actual Neto (VAN) como criterio fundamental de evaluación de negocios. Revista Economía y Desarrollo 128(1):180-194.

Chacón, A; Araya, Y; Gamboa, M. 2008. Percepciones y hábitos de consumo de la leche de cabra y sus derivados en los costarricenses. Agronomía Mesoamericana 19(2):241-250.

Corrales, J; Chacón, A. 2005. Estudio de opinión de consumidores sobre el queso fresco de cabra (Capra hircus) en Costa Rica. Revista de Agricultura Tropical 35:39-49.

HFAC (Humane Farm Animal Care). 2005. Estándares para el cuidado de los animales de granja. Estándar de cuidado de ganado ovino. Hendon, VA. USA. p. 6-10.
Luttmann, G. 1986. Raising milk goats succesfully. Nashville, Tennesse, USA. Williamson Editors. Chapters 2 y 4 . p. 21-27, 147-155.

Nastasi, V. 2007. Gestión Estratégica de los costos agropecuarios (en línea). Universidad de Buenos Aires, Argentina. Consultado 5 mayo 2009. Disponible en http://www.intercostos.org/documentos/Nastasi.pdf

Obando, G. 2005. Análisis de Rentabilidad en sistemas de producción lechera en ganadería de altura: Caso Finca Las Josefinas (en línea). IX Seminario de Pastos y Forrajes 2005. Consultado 11 set. 2009. Disponible en http://avpa.ula.ve/eventos/ix_seminario_pastosyforraje/Talleres/T4-GuidoObando.pdf

Sabadzija, GN; González, MF. 2007. Análisis económico de un modelo de producción de leche caprina en sistema intensivo bajo riego, en Catamarca, Argentina (en línea). Catamarca, Argentina. Consultado 2 jun. 2009. Disponible en http://www.produccionbovina.com/produccion_caprina/produccion_caprina/87-sabadzija. htm

Simiane, M; Le Mens, P. s.f. Batiments en élevage caprin. Société de Presse et d'Edition Ovine et Caprine. París, France. p. 25-35.

Vilaboa, J; Diaz, P; Platas, DE. et al. 2006. Productividad y autonomía en sistemas de producción ovina: Dos propiedades emergentes de los agroecosistemas. INCI 31(1):37-44.

Velasco, R; Gonzales, J; Jahn, E. s.f. Producción de cabras lecheras. Capítulo 11. p. 172. 